Original Article

\title{
Comparisons of Surgery and/or Chemotherapy in the Treatment of Primary Pulmonary Mucosa-Associated Lymphoid Tissue Lymphoma
}

\author{
Bo Wang, MD, ${ }^{1}$ Chengwei Zhang, $\mathrm{PhD},{ }^{2}$ Bin Wang, MM, ${ }^{1}$ and Lianbin Zhang, $\mathrm{MM}^{1}$
}

\begin{abstract}
Background/Aims: To investigate the clinical features, imaging characteristics, treatment, and prognosis of primary pulmonary mucosa-associated lymphoid tissue (MALT) lymphoma.

Methods: We retrospectively analysed the clinical, imaging, and follow-up data of 13 patients (median age, 59 years; range, 21-67 years) with primary pulmonary MALT lymphoma.

Results: The main clinical manifestations were chest discomfort (six patients), cough (two), fever (two), chest pain (one), and no obvious symptoms (two). Six patients underwent surgery; three had postoperative chemotherapy; four had chemotherapy alone; and three only had symptomatic and supportive treatment. The follow-up duration was one to 11 years, with one patient lost to follow-up. Two patients died (two years and 11 years post-diagnosis). As of this report, the remaining 10 patients were alive with no disease progression.

Conclusions: Pulmonary MALT lymphoma has atypical clinical manifestations and non-specific imaging changes, and the diagnosis depends on a pathological examination. For patients with confined lesions for which conventional biopsy cannot be performed, surgical excision plays an important role in clarifying the diagnosis and obtaining good therapeutic results and a good prognosis.
\end{abstract}

Keywords: MALT, lymphoma, surgery

\section{Introduction}

Mucosa-associated lymphoid tissue (MALT) lymphoma refers to a low-grade malignant B-cell lymphoma derived from the gastrointestinal tract, respiratory tract, and other mucous membranes. Primary pulmonary MALT

${ }^{1}$ Department of Thoracic Surgery, Chinese PLA General Hospital, Beijing, China

${ }^{2}$ Department of Thoracic Surgery, General Hospital of Armed Police Forces, Beijing, China

Received: May 28, 2014; Accepted: June 24, 2014

Corresponding author: Lianbin Zhang, MM. Department of Thoracic Surgery, Chinese PLA General Hospital, Beijing 100853, China Email: Drzhang2014@yeah.net

*Bo Wang and Chengwei Zhang contribute equally as first authors. (C)2014 The Editorial Committee of Annals of Thoracic and Cardiovascular Surgery. All rights reserved. lymphoma has a low incidence, and there are only a few relevant reports. It can be easily misdiagnosed because of non-specific clinical symptoms and imaging examination results. This study retrospectively analysed the clinical data of 13 patients with pathologically confirmed primary pulmonary MALT lymphoma that were treated at the PLA General Hospital from April 2000 to July 2012; moreover, we explored the diagnosis, treatment, and role of surgery in the diagnosis and treatment of pulmonary MALT lymphoma.

\section{Subjects and Methods}

\section{Subjects}

We collected the clinical data of 13 patients with pulmonary MALT lymphoma that were treated at the PLA General Hospital from April 2000 to July 2012. The inclusion 
criteria were as follows: (1) patients had lung tissue specimens obtained, and their primary pulmonary MALT lymphoma was confirmed by pathology; (2) patients had a complete clinical report, including chest computed tomography $(\mathrm{CT})$, pathological findings, treatment record, and follow-up data.

\section{Methods}

The clinical data of the patients with pulmonary MALT lymphoma were collected from their medical records, and we performed follow-up of the patients via phone calls or at an outpatient clinic. The follow-up treatment, survival time, and progression-free survival were recorded. Progression-free survival refers to the time from the date of the pathologically confirmed diagnosis to the date of disease progression or last follow-up. Survival time refers to the time from the date of the pathologically confirmed diagnosis to the date of the patient's death or last follow-up. The time of death was recorded for deceased patients, and the cause of death was determined to rule out deaths that were not related to pulmonary MALT lymphoma.

\section{Results}

\section{Clinical data}

This study included a total of 13 patients, of whom eight were men and five were women, with a male to female ratio of 1.6:1. The patients' age of onset ranged from 21 to 67 years, with a median age of 59 years. The main clinical manifestations were as follows: chest discomfort (six patients), cough (two patients), fever (two patients), chest pain (one patient), and no obvious symptoms (two cases). Of the two patients who had no obvious symptoms, they were admitted to the hospital because of detection of a pulmonary shadow at a physical examination. For those patients who had a chest $\mathrm{CT}$, the following findings were revealed: a pulmonary consolidation shadow with an air bronchogram (three patients), patchy shadows (three patients), mass shadows (four patients), and nodular ground-glass opacity (four patients). Ten patients had bilateral pulmonary lesions, while four patients also had mediastinal lymph node enlargement and one patient also had a pleural effusion. The patients' diagnoses upon admission were lung cancer (four patients), pneumonia (three patients), tuberculosis (three patients), giant lymph node hyperplasia (one patient), and diffuse interstitial lung disease (one patient); however, only one patient was diagnosed with lymphoma. One of the 13 patients also had coexisting Sjogren's syndrome. The patients' diagnoses were confirmed by the following methods: pathological examination of the surgically removed lung lesions (six patients), biopsy via bronchoscopy (four patients), and CT-guided lung biopsy (three patients). Both the pathological findings and immunohistochemical results suggested primary pulmonary non-Hodgkin extranodal marginal zone B-cell lymphoma of MALT.

\section{Treatment and prognosis}

Six of the 13 patients underwent surgical resection of the lung tissue lesions, of which three patients had four cycles of postoperative adjuvant chemotherapy. The regimen was either CHOP or R-CHOP (cyclophosphamide, doxorubicin, vincristine, and prednisone/prednisolone, with or without rituximab). One patient could not tolerate the side effects of chemotherapy, and the regimen was changed to two cycles of a fludarabine and cyclophosphamide (FC) regimen after two cycles of the CHOP regimen. Four patients had chemotherapy alone, with a regimen of either CHOP or R-CHOP, but one patient switched to a mitozantrone, cytarabine, and etoposide (MAE) regimen because of tumour progression. Three patients only had symptomatic and supportive treatment. No patients received radiotherapy.

The patients were followed for 12 to 132 months, and the median follow-up duration was 5 years. One of the 13 patients was lost to follow-up, so the follow-up rate was $92.3 \%$ (12/13). One patient experienced disease progression, 4 years after diagnosis, and died 11 years after diagnosis. Further, one patient died because of chemotherapy side effects within 2 years of diagnosis. The remaining 10 patients were still alive with no disease progression as of this report (Tables $\mathbf{1}$ and 2).

\section{Discussion}

\section{Epidemiology}

The concept of MALT lymphoma was first proposed by Isaacson and Wright in 1983. ${ }^{1)}$ In 2000, WHO defined MALT as an independent type of B-cell lymphoma. Primary pulmonary lymphoma is very rare, and the most common histological type of MALT is the bronchial extranodal marginal zone B-cell lymphoma with a long disease course, slow disease progression, few systemic symptoms, and a low incidence rate. ${ }^{2)}$ In this study, the median age of onset was 59 years, and the age ranged from 21 to 67 years. There were nine patients over 50 years of age that accounted for $69 \%$ of the patients. This study was 
Table 1 Clinical data of $\mathbf{1 3}$ patients with pulmonary MALT lymphoma

\begin{tabular}{|c|c|}
\hline Characteristics & Patients n $(\%)$ \\
\hline Median age of onset (years) & 59 \\
\hline Gender (male/female) & $\begin{array}{l}8(61.5 \%) / \\
5(38.5 \%)\end{array}$ \\
\hline Risk factors & $10(76.9 \%)$ \\
\hline Smoking & $4(30.8 \%)$ \\
\hline Infection & $9(69.2 \%)$ \\
\hline \multicolumn{2}{|l|}{ Main symptoms } \\
\hline Chest discomfort & $6(46.2 \%)$ \\
\hline Fever & $2(15.4 \%)$ \\
\hline Cough & $2(15.4 \%)$ \\
\hline Chest pain & $1(7.7 \%)$ \\
\hline No obvious symptoms & $2(15.4 \%)$ \\
\hline \multicolumn{2}{|l|}{ Imaging findings } \\
\hline Pulmonary consolidation shadow & $3(23.1 \%)$ \\
\hline Patchy shadow & $3(23.1 \%)$ \\
\hline Mass shadow & $4(30.8 \%)$ \\
\hline Nodular ground-glass opacity & $4(30.8 \%)$ \\
\hline Mediastinal lymph node enlargement & $4(30.8 \%)$ \\
\hline \multicolumn{2}{|l|}{ Diagnosis upon admission } \\
\hline Lung cancer & $4(30.8 \%)$ \\
\hline Pulmonary infection & $3(23.1 \%)$ \\
\hline Pulmonary tuberculosis & $3(23.1 \%)$ \\
\hline Giant lymph node hyperplasia & $1(7.7 \%)$ \\
\hline Diffuse interstitial lung disease & $1(7.7 \%)$ \\
\hline Lymphoma & $1(7.7 \%)$ \\
\hline \multicolumn{2}{|l|}{ Diagnosis is confirmed by } \\
\hline Surgery & $6(46.2 \%)$ \\
\hline Bronchoscopic biopsy & $4(30.8 \%)$ \\
\hline CT-guided percutaneous lung biopsy & $3(23.1 \%)$ \\
\hline \multicolumn{2}{|l|}{ Immunohistochemistry } \\
\hline CD20 (+) & $13(100 \%)$ \\
\hline CD79a (+) & $11(84.6 \%)$ \\
\hline CD5 (-) & $13(100 \%)$ \\
\hline CD10 (-) & $13(100 \%)$ \\
\hline CD23 (-) & $12(92.3 \%)$ \\
\hline \multicolumn{2}{|l|}{ Treatment } \\
\hline Surgery & $3(23.1 \%)$ \\
\hline Surgery plus chemotherapy & $3(23.1 \%)$ \\
\hline Chemotherapy & $4(30.8 \%)$ \\
\hline Symptomatic treatment & $3(23.1 \%)$ \\
\hline \multicolumn{2}{|l|}{ Prognosis } \\
\hline Duration of follow-up (months) & $12-132$ \\
\hline Lost to follow-up & $1(7.7 \%)$ \\
\hline Tumour-free survival & $10(76.9 \%)$ \\
\hline Death caused by illness & $1(7.7 \%)$ \\
\hline Death caused by other reasons & $1(7.7 \%)$ \\
\hline
\end{tabular}

MALT: mucosa-associated lymphoid tissue; CT: computed tomography

consistent with a report that pulmonary MALT lymphoma has a slightly higher incidence in men than in women, is more common in persons over 50 years of age, and is rare in persons younger than 30 years of age. ${ }^{3)}$

\section{Aetiology}

The cause of the disease remains unknown. The incidence of gastric MALT lymphoma is closely related to a helicobacter pylori infection; however, the current belief is that the incidence of pulmonary MALT lymphoma may be associated with an infection or smoking. ${ }^{4)}$ In this study, 10 patients had a history of pulmonary infection, long-term smoking, or both that supports the related report. Another study found that some autoimmune diseases such as Sjogren's syndrome and Hashimoto's thyroiditis can promote the incidence of pulmonary MALT lymphoma, but the specific mechanism remains unclear. ${ }^{5)}$ In this study, one patient had MALT combined with Sjogren's syndrome.

\section{Clinical manifestations}

The main manifestations of the disease are chest discomfort, chest pain, respiratory symptoms such as a cough and expectoration and non-specific symptoms such as fever. In this study, only two patients (15.4\%) had no obvious discomfort and sought medical help because of detection of a pulmonary shadow upon a physical examination. The majority of pulmonary MALT lymphoma is confined to the primary lesion; however, it was reported that patients with pulmonary MALT lymphoma have simultaneous oesophageal, stomach, colon, pleural, or thymus involvement. ${ }^{6)}$ Therefore, gastroscopy is recommended to exclude the involvement of the mucous membrane in other regions, after the diagnosis of pulmonary MALT lymphoma is confirmed, especially in those patients with gastrointestinal symptoms or a combined helicobacter pylori infection. In the study patients, no mucous membrane involvement in other regions was found.

\section{Imaging features}

Chest CT is an important examination for the diagnosis of this disease. The imaging manifestations of MALT are complex, but not typical. They include a pulmonary consolidation shadow, nodular shadow, mass shadow, and ground-glass opacity, which may be revealed by a bronchogram.7) In some patients, mediastinal lymph node enlargement and a pleural effusion can be seen but can also increase the difficulty in differentiating pulmonary MALT lymphoma from pneumonia, tuberculosis, and lung cancer. ${ }^{8)}$ In this study, the imaging findings were consistent with that of those reported in medical literature. Pulmonary consolidation is now considered the most common imaging manifestation; in particular, pulmonary MALT lymphoma should be considered if pulmonary consolidation is revealed by a bronchogram. 
Table 2 Treatment and prognosis of 13 patients with pulmonary MALT lymphoma

\begin{tabular}{|c|c|c|c|c|}
\hline Number & Treatment & $\begin{array}{l}\text { Time to recurrence } \\
\text { (months) }\end{array}$ & $\begin{array}{l}\text { Results of } \\
\text { follow-up }\end{array}$ & $\begin{array}{c}\text { Duration of } \\
\text { follow-up (months) }\end{array}$ \\
\hline 1 & Surgery & & Lost to follow-up & 60 \\
\hline 2 & Surgery & & Survival & 130 \\
\hline 3 & Surgery & & Survival & 111 \\
\hline 4 & Surgery plus chemotherapy & & Survival & 110 \\
\hline 5 & Chemotherapy & 48 & Death & 132 \\
\hline 6 & Surgery plus chemotherapy & & Survival & 61 \\
\hline 7 & Chemotherapy & & Survival & 88 \\
\hline 8 & Symptomatic treatment & & Survival & 17 \\
\hline 9 & Surgery plus chemotherapy & & Survival & 44 \\
\hline 10 & Chemotherapy & & $\begin{array}{l}\text { Death due to adverse } \\
\text { effect of chemotherapy }\end{array}$ & 16 \\
\hline 11 & Symptomatic treatment & & Survival & 12 \\
\hline 12 & Symptomatic treatment & & Survival & 40 \\
\hline 13 & Chemotherapy & & Survival & 28 \\
\hline
\end{tabular}

MALT: mucosa-associated lymphoid tissue

\section{Diagnostic method}

A confirmed diagnosis of pulmonary MALT lymphoma is dependent upon a biopsy. Generally, tissue specimens can be obtained through a percutaneous lung biopsy, bronchoscopy, or surgical resection. Immunohistochemistry and molecular detection are of significant importance in the diagnosis and differential diagnosis of MALT lymphoma. Pulmonary MALT lymphoma cells are derived from pulmonary interstitial and bronchial submucosal lymphoid tissue, and the main characteristic is small lymphocytic cell infiltration. Immunohistochemical results include the following: monoclonal B cells, CD20+, CD79a Bcl-10-positive, CD3-, CD45RO-, CD5-, CD23-, cyclin D1-, CD10-, and Bcl-2-negative. ${ }^{9)}$ For all patients in this study, their diagnoses were confirmed by pathology, and their immunohistochemical results were in line with those reported in medical literature.

\section{Treatment and prognosis}

Treatment of pulmonary MALT lymphoma is same as that of the other non-Hodgkin's lymphomas and includes surgery, chemotherapy, or surgery combined with postoperative chemotherapy. The specific treatment regimen is chosen based on a patient's condition. ${ }^{10)}$ Vanden Eynden, et al. thought proactive surgery was required for patients with confined pulmonary MALT lymphoma, which can be completely removed. ${ }^{11)}$ However, most scholars still recommend chemotherapy as the primary treatment. CHOP is still the most commonly used chemotherapy regimen; in this study, the main chemotherapy regimens were R-CHOP and CHOP. Some new chemotherapy regimens have been proposed in recent years, such as bendamustine with rituximab; ${ }^{12)}$ the efficacy of this regimen is comparable to that of R-CHOP, and its side effects are relatively minor. This regimen has been increasingly used as a first-line chemotherapy regimen for the treatment of MALT lymphoma. As radiotherapy can cause injury such as radiation pneumonitis, its application is limited. Troch, et al. observed 11 patients with pulmonary MALT lymphoma that were not treated immediately and found signs of spontaneous tumour regression in six patients. ${ }^{13)}$ The updated National Comprehensive Cancer Network (NCCN) guidelines of 2010 recommend follow-up only for some patients with non-gastric MALT lymphoma. $\mathrm{Li}$, et al. thought for some patients with pulmonary MALT lymphoma that do not have any obvious clinical symptoms, especially those patients in whom chemotherapy may cause serious adverse reactions, immediate treatment may not be required, and periodic follow-up was the best choice. ${ }^{14)}$ In our study, disease progression only occurred in one patient, four years after diagnosis, and only one patient died because of chemotherapy side effects. The remaining 10 patients had progression-free survival; thus, we endorse the recommendation of Li, et al. However, for patients with confined pulmonary MALT lymphoma for which pathological specimens cannot be obtained using conventional methods, surgical resection is still the best option. This strategy may not only clarify the diagnosis, but also achieve good therapeutic results.

Pulmonary MALT lymphoma is an indolent lymphoma with a slow progression and good prognosis. Its 5-year survival rate is up to $90 \% .{ }^{15}$ ) During the follow-up of the present study, disease progression only occurred in one of the 13 patients, and a patient who had more than 
20 repeated chemotherapy cycles died 11 years after diagnosis. Another patient died within two years of diagnosis because of a severe infection secondary to chemotherapyinduced severe bone marrow suppression. No disease progression occurred in the remaining 10 patients who survived as of this report, with the exception of one patient who was lost to follow-up. Our results are consistent with those in medical literature.

Atypical clinical manifestations and non-specific imaging findings, coupled with lack of clinician awareness, make pulmonary MALT lymphoma easy to misdiagnose as pulmonary inflammation, lung cancer, or tuberculosis. For patients with suspected pulmonary inflammation, lung cancer, or tuberculosis that have poor therapeutic results, pulmonary MALT lymphoma should be considered, and a timely pathological biopsy should be performed. For patients in whom specimens cannot be obtained using conventional methods such as bronchoscopy or CT-guided lung biopsy, proactive biopsy via surgery or surgical resection should be performed to clarify the diagnosis and guide subsequent treatment. Chemotherapy based on a CHOP regimen is the most important treatment for pulmonary MALT lymphoma, and rituximab in combination with chemotherapy has also been more widely used. Surgical treatment can achieve good results in patients with solitary and confined pulmonary MALT lymphoma. The prognosis of this disease is generally good, and the five-year survival rate is high. ${ }^{16)}$

\section{Disclosure Statement}

There is no conflict of interest.

\section{References}

1) Isaacson $\mathrm{P}$, Wright $\mathrm{DH}$. Malignant lymphoma of mucosa-associated lymphoid tissue. A distinctive type of B-cell lymphoma. Cancer 1983; 52: 1410-6.

2) Kocatürk Cİ, Seyhan EC, Günlüoğlu MZ, et al. Primary pulmonary non-Hodgkin's lymphoma: ten cases with a review of the literature. Tuberk Toraks 2012; 60: 246-53.

3) Cadranel J, Wislez M, Antoine M. Primary pulmonary lymphoma. Eur Respir J 2002; 20: 750-62.
4) Chanudet E, Adam P, Nicholson AG, et al. Chlamydiae and Mycoplasma infections in pulmonary MALT lymphoma. Br J Cancer 2007; 97: 949-51.

5) Ekström Smedby K, Vajdic CM, Falster M, et al. Autoimmune disorders and risk of non-Hodgkin lymphoma subtypes: a pooled analysis within the InterLymph Consortium. Blood 2008; 111: 4029-38.

6) Thieblemont C, Berger F, Dumontet C, et al. Mucosa-associated lymphoid tissue lymphoma is a disseminated disease in one third of 158 patients analyzed. Blood 2000; 95: 802-6.

7) Sui $X$, Song W, Jin ZY, et al. Clinical and imaging manifestations of pulmonary mucosa-associated lymphoid tissue lymphoma. Zhongguo Yi Xue Ke Xue Yuan Xue Bao 2012; 34: 41-5. (in Chinese)

8) King LJ, Padley SP, Wotherspoon AC, et al. Pulmonary MALT lymphoma: imaging findings in 24 cases. Eur Radiol 2000; 10: 1932-8.

9) Niu D, Sun K, Chen Y. Primary pulmonary lymphoma of mucosa-associated lymphoid tissue: a clinicopathological analysis of 4 cases. J Mod Oncol 2011; 19: 2519-21. (in Chinese)

10) Michael CW, Richardson PH, Boudreaux CW. Pulmonary lymphoma of the mucosa-associated lymphoid tissue type: report of a case with cytological, histological, immunophenotypical correlation, and review of the literature. Ann Diagn Pathol 2005; 9: 148-52.

11) Vanden Eynden F, Fadel E, de Perrot M, et al. Role of surgery in the treatment of primary pulmonary B-cell lymphoma. Ann Thorac Surg 2007; 83: 236-40.

12) Wang J, Wei L. Treatment progress in common indolent B-cell lymphoma: the 35th Congress of the European Society for Medical Oncology meeting report. J Leuk Lymph 2011; 20: 8-10. (in Chinese)

13) Troch M, Streubel B, Petkov V, et al. Does MALT lymphoma of the lung require immediate treatment? An analysis of 11 untreated cases with long-term follow-up. Anticancer Res 2007; 27: 3633-7.

14) Li L, Li X, Wen F. Clinical features and prognostic factors of pulmonary mucosa-associated lymphoid tissue lymphoma. Chin J Clin Oncol 2012; 39: 149-52. (in Chinese)

15) Li AW, Xu JF, Zhou CC, et al. Clinical characteristics and diagnosis of pulmonary mucosa-associated lymphoid tissue-derived (MALT) lymphoma: a retrospective analysis of 29 cases. Zhonghua Zhong Liu Za Zhi 2012; 34: 390-3. (in Chinese)

16) Borie R, Wislez M, Thabut G, et al. Clinical characteristics and prognostic factors of pulmonary MALT lymphoma. Eur Respir J 2009; 34: 1408-16. 